Claremont Colleges

Scholarship@ Claremont

All HMC Faculty Publications and Research

HMC Faculty Scholarship

$1-1-1998$

\title{
Positive Solutions for a Concave Semipositone Dirichlet Problem
}

\author{
Alfonso Castro \\ Harvey Mudd College \\ Ratnasingham Shivaji \\ Mississippi State University
}

\section{Recommended Citation}

Castro, Alfonso and Ratnasingham Shivaji. "Positive solutions for a concave semipositone Dirichlet problem”, Nonlinear Analysis TMA, Vol. 31, No. 1-2 (1998), pp. 91-98.

This Article - postprint is brought to you for free and open access by the HMC Faculty Scholarship at Scholarship @ Claremont. It has been accepted for inclusion in All HMC Faculty Publications and Research by an authorized administrator of Scholarship @ Claremont. For more information, please contact scholarship@cuc.claremont.edu. 


\section{PII: S0362-546X(96)00189-7}

\section{POSITIVE SOLUTIONS FOR A CONCAVE SEMIPOSITONE DIRICHLET PROBLEM $\dagger$}

\section{ALFONSO CASTRO $\ddagger$ and R. SHIVAJI\&}

$\ddagger$ Department of Mathematics, University of North Texas, Denton, TX 76203-5116, U.S.A.; and §Department of Mathematics and Statistics, Mississippi State University, Mississippi State, MS 39762, U.S.A.

(Received 31 August 1995; received in revised form 8 July 1996; received for publication 24 October 1996)

Key words and phrases: Semipositone, radial solutions, Pohozaev identity, uniqueness.

\section{INTRODUCTION}

We study the boundary value problem

$$
\begin{gathered}
-\Delta u(x)=\lambda f(u), \quad x \in \Omega ; \\
u(x)=0, \quad x \in \partial \Omega ;
\end{gathered}
$$

where $\Delta$ is the Laplacian operator, $\Omega$ is the unit ball in $\mathbb{R}^{N}, N \geq 1, f:[0, \infty) \rightarrow \mathbb{R}$ is a concave smooth function such that

$$
f(0)<0 \text {. }
$$

Nonnegative solutions to (1.1)-(1.2) are known to be positive and radial (see [1]). Also for (1.1)-(1.2) to have a positive solution it is necessary and sufficient that

$$
\int_{0}^{\alpha} f(s) \mathrm{d} s>0 \quad \text { for some } \alpha>0 .
$$

In fact, the results of [2] and [3] show that for any smooth bounded region the equations (1.1)-(1.2) have positive solutions for $\lambda>0$ large if (1.4) holds and

$$
\lim _{u \rightarrow \infty} f^{\prime}(u) \leq 0 .
$$

On the other hand, since positive solutions to $(1.1)-(1.2)$ are radial then $v(r)=u(x)$ for $\|x\|=r$ satisfies

$$
v^{\prime \prime}+\frac{N-1}{r} v^{\prime}+\lambda f(v)=0, \quad r \in[0,1] \quad u^{\prime}(0)=u(1)=0 .
$$

Letting $F(t)=\int_{0}^{t} f(s) \mathrm{d} s$, we see that $\left.E(r) \equiv\left(v^{\prime}(r)\right)^{2} / 2+F(v(r))\right)$ satisfies

$$
E^{\prime}(r)=-\frac{n}{r}\left(v^{\prime}(r)\right)^{2} \leq 0 .
$$

Since $E(1) \geq 0$ we have that either $E(0)=F(u(0))>0$ or $v$ is a constant function. Since no constant function satisfies (1.6) we conclude that (1.4) is also a necessary condition for (1.1)-(1.2) to have a solution.

\footnotetext{
† Partially supported by NSF Grant DMS-9215027.
} 


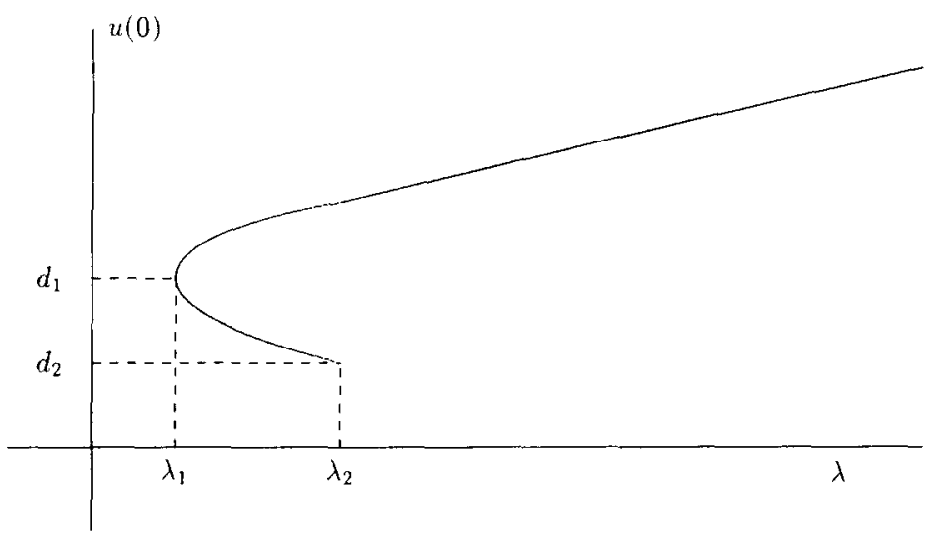

Fig. 1.

The rest of the article is devoted to showing that if (1.4)-(1.5) hold then the set of positive solutions to (1.1)-(1.2) corresponds to Fig. 1. That is, we prove the following result.

THEOREM 1.1. If (1.4)-(1.5) hold then the set of nonnegative solutions to (1.1)-(1.2) is connected and, for each $\lambda>0$ it has at most one stable solution and one unstable solution. There exist positive numbers $0<\lambda_{1}<\lambda_{2}$ such that: (1.1)-(1.2) has a stable solution if and only if $\lambda>\lambda_{1}$, and (1.1)-(1.2) has an unstable solution if and only if $\lambda \in\left[\lambda_{1}, \lambda_{2}\right]$.

Since Theorem 1.1 was proven in [4] when $\lim _{u \rightarrow \infty} f^{\prime}(0)=0$ we restrict ourselves in this paper to the case where $f$ has a second positive zero. That is, we assume $f$ to be like in Fig. 2, where $F(\theta)=0$ and $f^{\prime}(\tau)=0$. We note that without loss of generality we can assume that $\theta>\tau$. A key piece in the proof of Theorem 1.1 is showing that, for $\lambda>0$ large, (1.1)(1.2) has a unique positive solution. When $\lim _{u \rightarrow \infty} f^{\prime}(u)=0$, this proof was given in [5].

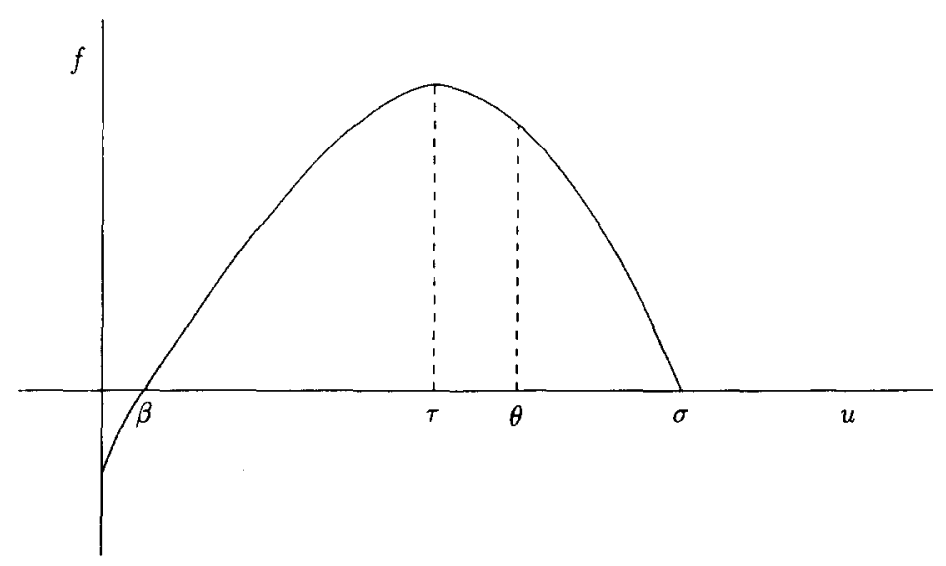

Fig. 2. 
In this case it is shown that solutions to (1.1)-(1.2) satisfy $\left|u^{\prime}(r)\right|=O(\lambda)$ for $r$ close to 1 , which is not valid when $\lim _{u \rightarrow \infty} f^{\prime}(u)<0$. In the latter case $\left|u^{\prime}(r)\right| \leq O\left(\lambda^{1 / 2}\right)$ for all $r \in[0,1]$.

Equations such as (1.1)-(1.2) arise, for example, in population models in the presence of "harvesting".

\section{A PRIORI ESTIMATES}

The arguments of this, and the ncxt, section hold under assumptions weaker than concavity. For the sake of simplicity we assume that

$$
f^{\prime}>0 \text { on }[0, \tau) \quad \text { and } \quad f^{\prime}<0 \text { on }(\tau, \sigma] .
$$

Let $(\lambda, u)$ be a positive solution to (1.1)-(1.2). Since $u$ is radial we have

$$
u^{\prime \prime}+\frac{N-1}{r} u^{\prime}+\lambda f(u)=0, \quad r \in[0,1] \quad u^{\prime}(0)=u(1)=0 .
$$

We now prove the following.

LEMMa 2.1. There exist positive constants $c$ and $\bar{\lambda}$ such that if $u$ is a positive solution to (2.2) then $\left(u^{\prime}(r)\right)^{2} \geq c \lambda$ if $u(r) \leq \tau$ and $\lambda>\bar{\lambda}$.

Since $u$ is positive $u(0)>\beta$. Let $r_{0} \in(0,1)$ be such that $u\left(r_{0}\right)=\beta / 2$ and $u(r) \leq \beta / 2$ for all $r \in\left[r_{0}, 1\right]$. Multiplying the second order differential equation in (2.2) by $r^{N-1}$ and integrating on $[r, 1]$ we have

$$
r^{N-1} u^{\prime}(r)=u^{\prime}(1)+\lambda \int_{r}^{1} s^{N-1} f(u(s)) \mathrm{d} s \leq \frac{\lambda f(\beta / 2)}{N}\left(1-r^{N}\right)
$$

for $r \in\left[r_{0}, 1\right]$. Since $r \leq 1$ we have $u^{\prime}(r) \leq \lambda f(\beta / 2)(1-r)$. Integrating on $\left[r_{0}, 1\right]$ we have $-\beta \leq \lambda f(\beta / 2)\left(1-r_{0}\right)^{2}$. Thus

$$
\left(1 \quad r_{0}\right)^{2} \leq \frac{-\beta}{\lambda f(\beta / 2)}
$$

Therefore, there exists $r_{1} \in\left[r_{0}, 1\right]$ such that

$$
u^{\prime}\left(r_{1}\right) \leq-(-\beta \lambda f(\beta / 2))^{1 / 2} / 2 \equiv-c_{1} \lambda^{1 / 2} .
$$

Let $r_{2}<r_{1}$ be such that $u\left(r_{2}\right)=\beta$. Arguing as above for $r \in\left[r_{2}, r_{1}\right]$ we have

$$
r^{N-1} u^{\prime}(r)=r_{1}^{N-1} u^{\prime}\left(r_{1}\right)+\lambda \int_{r}^{r_{1}} s^{N-1} f(u(s)) \mathrm{d} s \leq-r_{1}^{N-1} c_{1} \lambda^{1 / 2} .
$$

Integration of the latter inequality on $\left[r_{2}, r_{1}\right]$ yields

$$
-\beta \leq-r_{1}^{N-1} c_{1} \lambda^{1 / 2} \int_{r_{2}}^{r_{1}} s^{1-N} \mathrm{~d} s \leq-c_{1} \lambda^{1 / 2}\left(r_{1}-r_{2}\right) .
$$

Hence

$$
r_{1}-r_{2} \leq \frac{\beta}{c_{1} \lambda^{1 / 2}}
$$


Now replacing in (2.6) $r_{1}$ by $r_{2}$, using (2.6) and that $f$ attains its maximum value at $\tau$ we conclude

$$
\begin{aligned}
r^{N-1} u^{\prime}(r) & =r_{2}^{N-1} u^{\prime}\left(r_{2}\right)+\lambda \int_{r}^{r_{2}} s^{N-1} f(u(s)) \mathrm{d} s \\
& \leq-r_{2}^{N-1} c_{1} \lambda^{1 / 2}+\frac{\lambda f(\tau)}{N}\left(r_{2}^{N}-r^{N}\right),
\end{aligned}
$$

for $r \leq r_{2}$. Since $\left(r_{2}^{N}-r^{N}\right) \leq N r_{2}^{N-1}\left(r_{2}-r\right)$, from (2.9) we see that for $r_{2}-r \leq c_{1} / 2 f(\tau) \lambda^{1 / 2}$

$$
u^{\prime}(r) \leq-\left(c_{1} / 2\right) \lambda^{1 / 2}
$$

Let $r_{3} \equiv r_{2}-c_{1} / 2 f(\tau) \lambda^{1 / 2}$. Integrating on $\left[r_{3}, r_{2}\right]$ we have

$$
\sigma>u\left(r_{3}\right) \geq \beta+\frac{c_{1}^{2}}{4 f(\tau)} .
$$

Since (1.5) implies that $f$ is bounded, and since $E(r) \leq E(0)=\lambda F(u(0))$ for all $r \in[0,1]$, we have

$$
\left|u^{\prime}(r)\right| \leq c_{2} \lambda^{1 / 2} \quad \text { for all } r \in[0,1] .
$$

Let $\tau_{1} \geq(\theta+\sigma) / 2>\tau>\tau_{0}>\beta$ be such that $f\left(\tau_{0}\right)=f\left(\tau_{1}\right)=\min (f((\theta+\sigma) / 2)$, $\left.f\left(\beta+c_{1}^{2} / 4 f(\tau)\right)\right\}$. Let $0<r_{5}<r_{4}<1$ be such that $u\left(r_{5}\right)=\tau_{1}$ and $u\left(r_{4}\right)=\tau_{0}$. Note that, by (2.11), $r_{4}>r_{3}$. Since $f(u(s)) \geq f\left(\tau_{0}\right)$ for all $s \in\left[r_{5}, r_{4}\right]$, we have

$$
\begin{aligned}
r_{4}^{N-1} u^{\prime}\left(r_{4}\right) & =r_{5}^{N-1} u^{\prime}\left(r_{5}\right)-\lambda \int_{r_{5}}^{r_{4}} s^{N-1} f(u(s)) \mathrm{d} s \\
& \leq-\frac{f\left(\tau_{0}\right) \lambda}{N}\left(r_{4}-r_{5}\right)\left(r_{4}^{N-1}+\cdots+r_{5}^{N-1}\right) .
\end{aligned}
$$

This and (2.12) imply

$$
r_{5} \geq r_{4}-\frac{c_{2} N}{\lambda^{1 / 2} f\left(\tau_{0}\right)}
$$

Now using (2.4), (2.8), (2.13), $r_{1} \geq r_{0}, r_{4}>r_{3}$ we conclude

$$
\begin{aligned}
r_{5} & \geq r_{3}-\frac{c_{2} N}{\lambda^{1 / 2} f\left(\tau_{0}\right)} \\
& =r_{2}-\left(\frac{c_{1}}{2 f(\tau)}+\frac{c_{2} N}{f\left(\tau_{0}\right)}\right) \lambda^{-1 / 2} \\
& \geq r_{1}-\left(\frac{\beta}{c_{1}}+\frac{c_{1}}{2 f(\tau)}+\frac{c_{2} N}{f\left(\tau_{0}\right)}\right) \lambda^{-1 / 2} \\
& \geq 1-\left(\frac{\beta}{c_{1}}+\frac{c_{1}}{2 f(\tau)}+\frac{c_{2} N}{f\left(\tau_{0}\right)}+\left(\frac{-\beta}{f(\beta / 2)}\right)^{1 / 2}\right) \lambda^{-1 / 2} \\
& \equiv 1-c_{3} \lambda^{-1 / 2}
\end{aligned}
$$


Finally we estimate $\left|u^{\prime}(r)\right|$ for $u(r) \leq \tau$. From the definition of $E$, for $\lambda$ sufficiently large and $u(r) \leq \tau$, we have

$$
\begin{aligned}
\left(u^{\prime}(r)\right)^{2} & =\left(u^{\prime}\left(r_{5}\right)\right)^{2}+2 \lambda\left(F\left(u\left(r_{5}\right)\right)-F(u(r))\right)-2 \int_{r_{5}}^{r} \frac{n\left(u^{\prime}(s)\right)^{2}}{s} \mathrm{~d} s \\
& \geq 2 \lambda\left(F\left(\frac{\theta+\sigma}{2}\right)-F(\tau)\right)-\frac{\left(1-r_{5}\right) 2 n\left(c_{2}\right)^{2} \lambda}{r_{5}} \\
& \geq\left(F\left(\frac{\theta+\sigma}{2}\right)-F(\tau)\right) \lambda \equiv c \lambda
\end{aligned}
$$

where we have used (2.14) to estimate $1-r_{5}$ and that $r \geq r_{5}$ for $u(r) \leq \tau$.

\section{UNIQUENESS OF POSITIVE SOLUTIONS FOR $\lambda$ LARGE}

By the maximum principle, and because $f(\sigma)=0$, we see that if (1.1)-(1.2) has a positive solution than it has a maximal positive solution. Thus if we assume that (1.1)-(1.2) has two positive solutions $u$ and $v$, without loss of generality, we can assume that $v(x)>u(x)$ for all $x \in \Omega$. We let $z \equiv v-u$, and $s_{1}, s_{2}, s_{3} \in(0,1)$ such that $u\left(s_{1}\right)=\tau_{1}$, $u\left(s_{2}\right)=\tau=v\left(s_{3}\right)$. Since $u$ and $v$ are radially decreasing we have $s_{1}<s_{2}<s_{3}$. By (2.1) and the definition of $s_{2}$ we have $f(v(s))-f(u(s)) \leq 0$ for all $s \in\left[0, s_{2}\right]$. Hence, letting $z \equiv v-u$ we have

$$
t^{N-1} z^{\prime}(t)=-\lambda \int_{0}^{t} s^{N-1}(f(v(s))-f(u(s))) \mathrm{d} s \geq 0
$$

for all $t \in\left[0, s_{2}\right]$. Since $z^{\prime}(1) \leq 0$ we have

$$
\int_{0}^{s_{1}} s^{N-1}(f(u(s))-f(v(s))) \mathrm{d} s \leq \int_{s_{1}}^{1} s^{N-1}(f(v(s))-f(u(s))) \mathrm{d} s .
$$

This, (2.14), and (3.1) imply

$$
\begin{aligned}
k_{1} \int_{0}^{s_{1}} s^{N-1}(v(s)-u(s)) \mathrm{d} s & \leq \int_{0}^{s_{1}} s^{N-1}(f(u(s))-f(v(s))) \mathrm{d} s \\
& \leq \int_{s_{1}}^{1} s^{N-1}(f(v(s))-f(u(s))) \mathrm{d} s \\
& \leq c_{3} k_{2} \lambda^{-1 / 2} \max \left\{z(s) ; s \in\left[s_{1}, 1\right]\right\} \\
& \leq c_{3} k_{2} \lambda^{-1 / 2} \max \left\{z(s) ; s \in\left[s_{2}, 1\right]\right\}
\end{aligned}
$$

where $k_{1}=\min \left\{-f^{\prime}(t) ; t \in\left[\tau_{1}, \sigma\right]\right\}$, and $k_{2}=\max \left\{\left|f^{\prime}(t)\right| ; t \in[0, \sigma]\right\}$. Now we are ready to prove the uniqueness of positive solutions for $(1.1)-(1.2)$ when $\lambda$ is large.

THEOREM 3.1. There exists $\lambda_{0}>0$ such that if $\lambda>\lambda_{0}$ equation (1.1)-(1.2) has a unique positive solution. 
Proof. Recalling the Pohozaev identity (see [5]) we see that

$$
\left(u^{\prime}(1)\right)^{2}=2 \lambda \int_{0}^{1} s^{N-1} P(u(s)) \mathrm{d} s,
$$

where $P(t)=N \int_{0}^{t} f(s) \mathrm{d} s-((N-2) / 2) t f(t)$. Similarly for $\left(v^{\prime}(1)\right)^{2}$. This, (2.15) and (3.2) give

$$
\begin{aligned}
\left|z^{\prime}(1)\right| & =\frac{2 \lambda \int_{0}^{1} s^{N-1}(P(v(s))-P(u(s))) \mathrm{d} s}{\left|u^{\prime}(1)+v^{\prime}(1)\right|} \\
& \leq\left(\frac{\lambda}{c}\right)^{1 / 2} k_{3} \int_{s_{1}}^{1} s^{N-1}\left(v(s)-u(s)+\frac{f(v(s))-f(u(s))}{k_{1}}\right) \mathrm{d} s \\
& \leq\left(\frac{\lambda}{c}\right)^{1 / 2} k_{3} \int_{s_{1}}^{1} s^{N-1}(v(s)-u(s))\left(1+\frac{k_{2}}{k_{1}}\right) \mathrm{d} s \\
& \leq\left(1+\frac{k_{2}}{k_{1}}\right)\left(\frac{1}{c}\right)^{1 / 2} k_{3} c_{3} \max \left\{z(s) ; s \in\left[s_{2}, 1\right]\right\} \\
& \equiv k_{4} \max \left[z(s) ; s \in\left[s_{2}, 1\right]\right\},
\end{aligned}
$$

where $k_{3}=\max \left\{\left|P^{\prime}(t)\right| ; t \in[0, \sigma]\right\}$. Because $f(v(s))-f(u(s))>0$ for $s \in\left[s_{3}, 1\right]$ we have

$$
s^{N-1} z^{\prime}(s)=z^{\prime}(1)+\lambda \int_{s}^{1} s^{N-1}\left(f(v(s))-f(u(s)) \mathrm{d} s \geq z^{\prime}(1) .\right.
$$

Since for $\lambda$ large $s_{3}^{N-1}>1 / 2$ [see (2.14)], we have $z^{\prime}(s) \geq-2 k_{4} \max \left\{z(s) ; s \in\left[s_{2}, 1\right]\right\}$ for all $s \in\left[s_{3}, 1\right]$. In particular, by (2.14) we have

$$
z(t) \leq 2 k_{4} \max \left[z(s) ; s \in\left[s_{2}, 1\right]\right\} c_{3} \lambda^{-1 / 2},
$$

for all $t \in\left[s_{3}, 1\right]$. Since this implies that $u\left(s_{3}\right) \geq \tau-2 k_{4} \max \left\{z(s) ; s \in\left[s_{2}, 1\right]\right\} c_{3} \lambda^{-1 / 2}$, by (2.15) we have $s_{2} \geq s_{3}-2 k_{4} \max \left\{z(s) ; s \in\left[s_{2}, 1\right]\right\} c_{3} \lambda^{-1} / c^{1 / 2}$. Now for $t \in\left[s_{2}, s_{3}\right]$ we have

$$
\begin{aligned}
t^{N-1} z^{\prime}(t) & =s_{3}^{N-1} z^{\prime}\left(s_{3}\right)+\lambda \int_{t}^{s_{3}} s^{N-1}(f(v(s))-f(u(s))) \mathrm{d} s \\
& \geq-\max \left\{z(s) ; s \in\left[s_{2}, 1\right]\right\}\left(2 k_{4}+\frac{(f(\tau)-f(0)) 2 k_{4} c_{3}}{c^{1 / 2}}\right) .
\end{aligned}
$$

Thus for $\lambda$ sufficiently large we have

$$
z^{\prime}(t) \geq-2 \max \left\{z(s) ; s \in\left[s_{2}, 1\right]\right\}\left(2 k_{4}+\frac{(f(\tau)-f(0)) 2 k_{4} c_{3}}{c^{1 / 2}}\right),
$$

for $t \in\left[s_{2}, s_{3}\right]$. Now for $t \in\left[s_{2}, 1\right]$ and $\lambda$ sufficiently large, by (3.5) we have

$$
\begin{aligned}
z(t) & \leq 2 c_{3} \lambda^{-1 / 2} \max \left\{z(s) ; s \in\left[s_{2}, 1\right]\right\}\left(2 k_{4}+\frac{(f(\tau)-f(0)) 2 k_{4} c_{3}}{c^{1 / 2}}\right) \\
& \leq(1 / 2) \max \left\{z(s) ; s \in\left[s_{2}, 1\right]\right\} .
\end{aligned}
$$

Since (3.7) implies that $z=0$ on $\left[s_{2}, 1\right]$, by uniqueness of solutions to initial value problems we see that $v=u$, which concludes the proof. 


\section{PROOF OF THEOREM 1.1}

Here we use extensively the developments of [4].

For $\lambda>0, d>0$, let $u(\cdot, \lambda, d)$ denote the solution to the initial value problem

$$
u^{\prime \prime}+\frac{N-1}{r} u^{\prime}+\lambda f(u)=0 \quad r \in[0,1], u^{\prime}(0)=0, u(0)=d
$$

Using the fact that $f$ is concave and $\lim _{u \rightarrow \infty} f^{\prime}(u) \leq 0$, it follows that the function $u(\cdot, \lambda, d)$ has the following properties:

(a) If $u(\cdot, \lambda, d)>0$ in $[0,1), u(1, \lambda, d)=0$, then $u_{d}(\cdot, \lambda, d)=0$ has at most one zero in $[0,1]$.

(b) If $u\left(\cdot, \lambda_{1}, d_{1}\right)>0$ in $[0,1), u\left(1, \lambda_{1}, d_{1}\right)=0$, and $u_{d}\left(1, \lambda_{1}, d_{1}\right)=0$ then $u_{\lambda}\left(1, \lambda_{1}, d_{1}\right)<0$ and $u_{d d}\left(1, \lambda_{1}, d_{1}\right)>0$. Moreover, there exists $\varepsilon>0$ and a differentiable function

$$
\Lambda:\left(d_{1}-\varepsilon, d_{1}+\varepsilon\right) \rightarrow R
$$

such that $u(1, \Lambda(d), d)=0, \Lambda^{\prime}\left(d_{1}\right)=0$ and $\Lambda^{\prime \prime}\left(d_{1}\right)>0$.

(c) If $\Gamma \subset R^{2}$ is a connected component of $\left\{(\lambda, d) ; u\left(\cdot, \lambda_{0}, d_{0}\right)>0\right.$ in $[0,1)$, $u\left(1, \lambda_{0}, d_{0}\right)=0$, then $\Gamma$ is unbounded in the $\lambda$ direction.

These properties were proved in Lemmas 1-3 in [4] in the case $\lim _{u \rightarrow \infty} f^{\prime}(u)=0$. However we point out that in Lemma 3 of [4] the proof misses the observation that for each $\mu>0$ there exists an $M(\mu)$ such that $\|u\|_{\infty} \leq M(\mu)$ for every $\lambda \in(0, \mu)$, which follows from $\lim _{u \rightarrow \infty} f^{\prime}(u)=0$. In the case $\lim _{u \rightarrow \infty} f^{\prime}(u)<0$ it is easy to observe that $M(\mu)=\sigma$. Further since there are no positive solutions for $\lambda>0$ small, we must have

(d) There exists $\left(\lambda_{1}, d_{1}\right) \in \Gamma$ such that $u_{d}\left(1, \lambda_{1}, d_{1}\right)=0$.

Now from Theorem 3.1 and (c) we see that

$$
\left\{(\lambda, d) ; u\left(\cdot, \lambda_{0}, d_{0}\right)>0 \text { in }[0,1], u\left(1, \lambda_{0}, d_{0}\right)=0\right\} \equiv \Gamma
$$

is connected. From (b) and the implicit function theorem we see that $\Gamma$ is a differentiable curve. Also, by the uniqueness of solutions to the initial value problem (4.1)

$$
u(r / \rho, \lambda, d)=u\left(r, \lambda / \rho^{2}, d\right), \quad \text { for any } \rho>0 .
$$

Suppose that $(\alpha, d) \in \Gamma$ and $(\beta, d) \in \Gamma$ with $\alpha<\beta$. Using (4.2) we see that $u\left((\alpha / \beta)^{1 / 2}, \beta, d\right)=0$. Hence for $\lambda=\beta$ equation (1.1)-(1.2) has a solution with interior zeros $(r=\alpha / \beta)$. Because nonnegative solutions to (1.1)-(1.2) are positive we have a contradiction. Thus for each $d>0$ there exists at most one $\lambda>0$ such that $(\lambda, d) \in \Gamma$. Therefore the function $\Lambda$ in (b) can be extended to $A=\{d ;(\lambda, d) \in$ for some $\lambda>0\}$. Also by (b) the number $d_{1}$ is unique, $\Lambda^{\prime}(d)>0$ for $d>d_{1}$, and $\Lambda^{\prime}(d)<0$ for $d<d_{1}$. Because nonnegative solutions to (1.1)-(1.2) satisfy $u(0)>\theta, d_{2}=\inf A \geq \theta$. Since, by Theorem 3.1, the problem (1.1)-(1.2) has a unique solution for $\lambda$ large, and because of results [2] for $\lambda>0$ large this problem has a stable solution, we conclude that for $\lambda>0$ large enough $u_{d}(1, \lambda, d)>0$. On the other hand since $\Lambda^{\prime}(d)<0$, for $d \in\left(d_{2}, d_{1}\right)$, we have $u_{d}(1, \Lambda(d), d)<0$, and by uniqueness for $\lambda$ large we have $\lim _{d \rightarrow d_{2}} \Lambda(d)<\infty$. Further, by continuous dependence of solutions to (4.1) in parameters we see that $\left(\lim _{d \rightarrow d_{2}} \Lambda(d), d\right) \in \Gamma$. Taking $\lambda_{2}=\lim _{d \rightarrow d_{2}} \Lambda(d)$ the theorem is proven. 


\section{REFERENCES}

1. Castro, A. and Shivaji, R., Non-negative solutions to a semilinear Dirichlet problem in a ball are positive and radially symmetric. Comm. Partial Differential Equations, 1989, 14, 1091-1100.

2. Clement, P. and Sweers, G., Existence and multiplicity results for a semilinear elliptic eigenvalue problem. Ann. Scuola Norm. Sup. Pisa. Cl. Sci., 1987, 14(4), 97-121.

3. Castro, A., Garner, J. and Shivaji, R., Existence results for classes of sublinear semipositone problems. Results in Mathematics, 1993, 23, 214-220.

4. Castro, A. and Gadam, S., Uniqueness of stable and unstable positive solutions for semipositone problems. Nonlinear Analysis T.M.A., 1994, 22, 425-429.

5. Ali, I., Castro, A. and Shivaji, R., Uniqueness and stability of nonnegative solutions for semipositone problems in a ball. Proceedings A.M.S., 1993, 117, 775-782. 